\title{
Tratamento da pitiose cutânea equina com acetato de triancinolona e iodeto de potássio: relato de casos
}

\author{
Treatment of equine cutaneous pythiosis with triamcinolone acetate and \\ potassium iodide: case reports
}

Gabriela Bravim Lemos*, Laura Bravo Defanti Venâncio Petrucci, Veronica Vieira, Paula Alessandra Di Filippo

Universidade Estadual do Norte Fluminense Darcy Ribeiro (UENF), Campos dos Goytacazes, RJ, Brasil

\section{Resumo}

Apesar da existência de inúmeras propostas de tratamento para a pitiose cutânea equina, ainda se faz necessária a descoberta e o estudo de novos protocolos terapêuticos. 0 objetivo deste estudo foi avaliar a eficácia do tratamento da pitiose cutânea equina com o uso do acetato de triancinolona, $50 \mathrm{mg} /$ animal, por via intramuscular (IM), a cada sete dias, em um total de três aplicações, associado ao iodeto de potássio, $10 \mathrm{~g} / \mathrm{animal}$, via oral (VO), a cada 24 horas (SID), por 15 dias. Os cinco animais possuíam escore corporal 4 e idade entre 4 meses e 10 anos, sendo quatro fêmeas e um macho castrado. A confirmação do diagnóstico da pitiose foi realizada com exame clínico e histopatologia. Os animais apresentavam lesões únicas. Todas possuíam de 30 a 60 dias de evolução. 0 tratamento resultou em melhora clínica completa, com remissão macroscópica das lesões, sem a necessidade de exérese cirúrgica, podendo ser considerado como uma boa alternativa no tratamento de pitiose equina.

Palavras-chave: Pythium insidiosum. Corticoesteróide. Inflamação.

\begin{abstract}
Despite the existence of numerous proposals for equine cutaneous pythiosis treatment, the discovery and study of new therapeutic protocols are still necessary. The objective of this study was to evaluate the effectiveness of using triamcinolone acetonide, $50 \mathrm{mg} /$ animal, intramuscular (IM), three injections with 7 days interval, associated with potassium iodide, $10 \mathrm{~g} /$ animal/SID, orally (VO), for 15 days. The five animals had body score of 4 and age between 4 months and 10 years old. There were four females and one gelding. The pythiosis diagnosis was reached with the clinical and histopathological examination. The lesions were unique and they had 30 to 60 days of evolution. The treatment presented efficacy, with macroscopic lesions remission and a complete cure with no need for surgical excision, being considered a good alternative in the equine pythiosis treatment.
\end{abstract}

Keywords: Corticosteroid. Inflammation. Pythium insidiosum. 


\section{Introdução}

A pitiose é uma doença piogranulomatosa de distribuição mundial, causada pelo fungo Pythium insidiosum, um zoósporo biflagelado que se reproduz em água estagnada. Ocorre principalmente em regiões de climas tropicais, como o Brasil, e acomete equinos de diferentes idades e de ambos os sexos. As lesões se caracterizam pela formação de granulomas, ulcerados e pela presença de massas necróticas amareladas e friáveis, conhecidas por kunkers. A doença é popularmente conhecida como "zigomicose", "ferida de verão", "ferida brava", "mal dos pântanos" ou "ferida da moda" (Santurio et al., 2006). Na região Norte Fluminense do estado do Rio de Janeiro, recebe o nome de formigueiro (Carvalho et al., 2000).

No Brasil, a pitiose é a terceira doença tegumentar mais frequentemente observada em cavalos, representando 9,44\% dessas enfermidades, como descrito em um estudo realizado no Rio Grande do Sul com 710 cavalos (Assis-Brasil et al., 2015). Em um período de 32 anos, a pitiose se constituiu na principal enfermidade cutânea dos equinos (Marcolongo-Pereira et al., 2012). Na região Norte Fluminense, no estado do Rio de Janeiro, 30 casos foram descritos durante dois anos (Viana et al., 2015), sendo que a confirmação diagnóstica da pitiose pode ser feita por meio de exame clínico associado ao histopatológico (Santos et al., 2011), e do teste de ELISA a partir do soro (Santurio et al., 2006).

Diferentes propostas de tratamento foram sugeridas ao longo dos anos, e os resultados se mostraram variáveis. A exérese cirúrgica da lesão cutânea pode ser utilizada isoladamente como uma das formas de tratamento, devendo haver remoção total daárea comprometida pelo pitium, ou associada a terapias orais e parenterais. Sucessos na remição da doença foram mencionados após o uso de iodeto de potássio (Salomão-Nascimento et al., 2010) e de imunoterápicos como o PitiumVac ${ }^{\circledR}$ (Biava et al., 2007). A perfusão regional com anfotericina $B$ associada ao dimetilsulfóxido (DMSO) e a exérese cirúrgica da lesão resultaram na cura dos animais avaliados (Dória et al., 2012). Recentemente, relatos do uso do acetato de triancinolona associado ao iodeto de potássio para tratamento da pitiose foram descritos (Cardona-Álvarez et al., 2017). Frente às alternativas terapêuticas para a doença e às diferentes respostas aos tratamentos, o objetivo deste estudo foi avaliar a eficácia do uso do acetato de triancinolona associado ao iodeto de potássio no tratamento da pitiose cutânea equina.

\section{Relato de caso}

No decorrer do ano de 2016, cinco equinos foram atendidos com diagnóstico de pitiose cutânea confirmado por meio do exame de histopatologia (hematoxilina e eosina - H/E - e Metenamina de prata de Grocott - GMS).

Os animais acometidos pelo pitium possuíam idade entre 4 meses e 10 anos, e consistiam em quatro fêmeas e um macho castrado. Os cinco animais apresentavam lesões únicas localizadas na região ventral do abdômen $(\mathrm{n}=1)$, no metacarpo $(\mathrm{n}=3)$ e no metatarso $(\mathrm{n}=1)$, com intervalo de evolução de 30 a 60 dias. As feridas possuíam de 10 a $15 \mathrm{~cm}$ de diâmetro (Tabela 1 ). Três animais de diferentes propriedades eram criados soltos a pasto e possuíam acesso a áreas alagadiças, entretanto dois animais eram criados exclusivamente em baias. Todos os proprietários relataram que as feridas não cicatrizavam, aumentavam rapidamente de tamanho e possuíam odor ruim. Clinicamente, as lesões apresentavam aspecto granulomatoso, com superfície ulcerada e presença de kunkers, drenavam secreção sero-sanguinolenta e apresentavam odor fétido.

Tabela 1 - Identificação e dados dos animais descritos no estudo

\begin{tabular}{cccccc}
\hline Animal & Sexo & $\begin{array}{c}\text { Idade } \\
\text { (meses) }\end{array}$ & $\begin{array}{c}\mathrm{N}^{0} \\
\text { lesões }\end{array}$ & Localização & $\begin{array}{c}\text { Evolução } \\
\text { (dias) }\end{array}$ \\
\hline 1 & fêmea & 96 & 1 & abdômen & 30 \\
2 & fêmea & 84 & 1 & metacarpo & 60 \\
3 & fêmea & 120 & 1 & metacarpo & 60 \\
4 & fêmea & 4 & 1 & metatarso & 40 \\
5 & macho & 60 & 1 & metacarpo & 30 \\
\hline
\end{tabular}

Todos os animais se apresentavam bem nutridos, com escore corporal 4, exceto a fêmea, que apresentava lesão abdominal e escore 3 . Todas 
as fêmeas adultas $(\mathrm{n}=3)$ foram submetidas ao exame ultrassonográfico e à palpação retal para diagnóstico de gestação, com resultado negativo. Os animais com feridas em membros também foram submetidos à avaliação radiográfica para analisar possível acometimento ósseo. Após o diagnóstico confirmado de pitose, os animais foram submetidos ao tratamento com acetato de triancinolona. 0 medicamento foi administrado na dosagem de 50 $\mathrm{mg}$ /animal, por via intramuscular (IM), a cada sete dias, em um total de três aplicações, e iodeto de potássio na dose de $10 \mathrm{~g} /$ animal, por via oral (VO) a cada 24 horas (SID), por 15 dias. Não foi realizada a exérese cirúrgica das lesões cutâneas. Para evitar a contaminação com sujidades e a automutilação dos animais com lesões nos membros, foram feitas bandagens com vaselina sólida, compressa estéril, algodão e ataduras. A remissão total da afecção, incluindo o fechamento das lesões cutâneas, foi observada com aproximadamente
70 dias. Os kunkers e a presença de secreção serosanguinolenta, entretanto, apresentaram remissão após 20 dias de tratamento.

\section{Resultados e discussão}

Os cinco animais, medicados com acetato de triancinolona e iodeto de potássio, apresentaram resposta positiva ao tratamento. As lesões acometeram, em sua maioria, os membros locomotores (Figura 1), com exceção de uma fêmea, em que a lesão acometia a parte ventral do abdômen (Figura 2), próxima à cicatriz umbilical. Dentre os animais, encontravam-se exemplares do sexo masculino e feminino, adultos e jovens, de diferentes raças e sem raça definida, o que nos permite ressaltar que a doença não possui predileção por essas características, corroborando com as descrições de Miller e Campbell (1982).
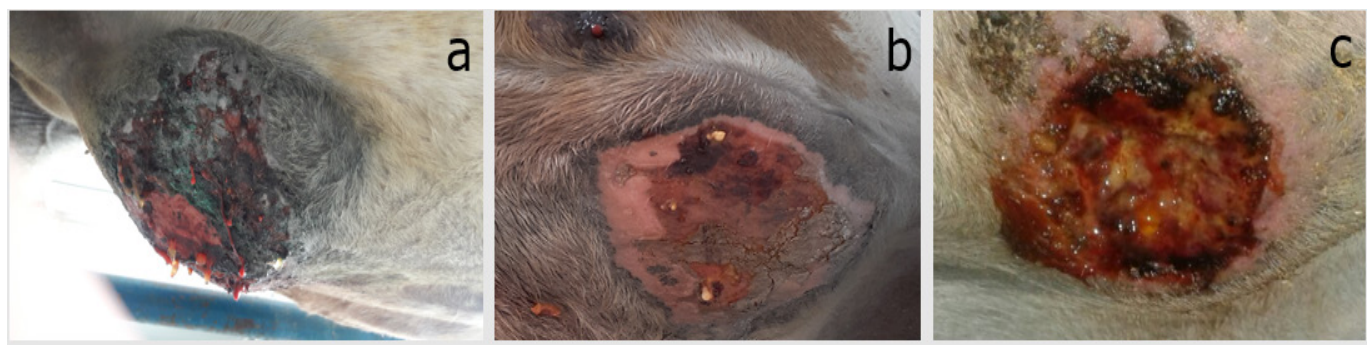

Figura 1 - (a) Ferida abdominal da fêmea equina antes do tratamento proposto. (b) Ferida após 10 dias do tratamento inicial, evidenciando a presença de kunkers. (c) Ferida após 15 dias de tratamento.
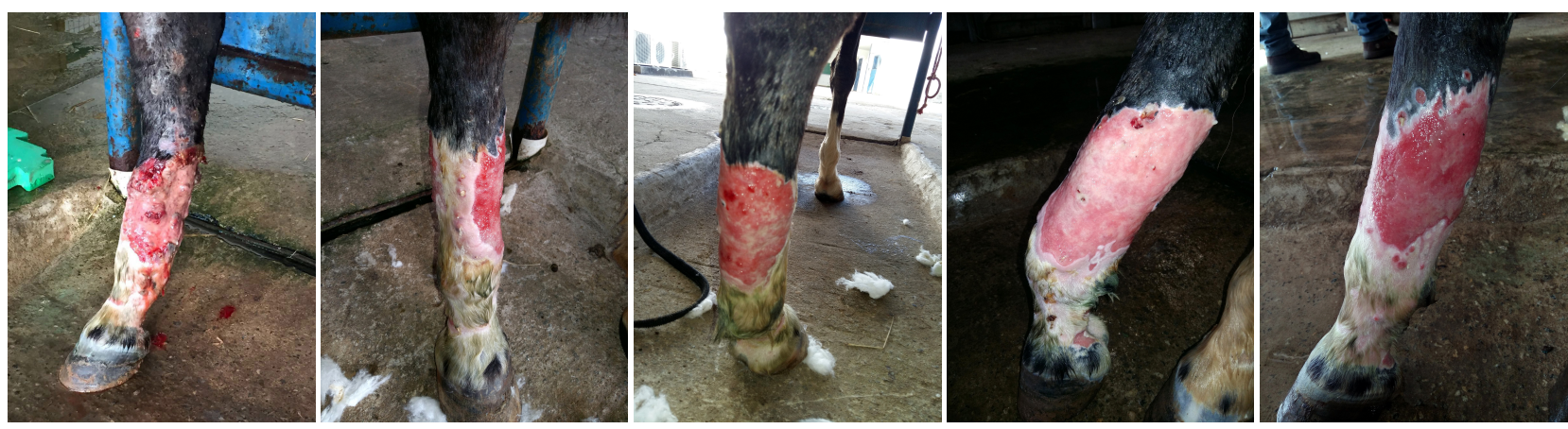

Figura 2 - Feridas no membro posterior direito da fêmea equina com 120 meses de idade. Da esquerda para a direita, as imagens indicam a evolução da mesma ferida após o início do tratamento. 
A alta incidência de pitiose localizada nos membros locomotores pode estar associada ao manejo e ao acesso dos equinos a ambientes úmidos. O contato das extremidades distais com a água parada e seus detritos faz com que os zoósporos atinjam, por quimiotaxia, pequenas lesões cutâneas presentes na pele dos animais (Costa et al., 2012). Os equinos soltos a pasto possuem o hábito de andar enquanto buscam alimento, sendo comum encontrar animais pastando nas margens dos rios ou até mesmo dentro do curso d'água, favorecendo o surgimento da afecção principalmente na porção distal dos membros e na região tóracoabdominal ventral (Sallis et al., 2003; Santos et al., 2011). Frequentemente, também pode ocorrer o acometimento da face (Mosbah et al., 2012). Nas diferentes espécies suscetíveis à pitiose, já foi descrito o acometimento de ossos, linfonodos, olhos, artérias e órgãos dos sistemas digestivo e respiratório (Santurio et al., 2004).

Dois dos animais do presente estudo viviam em baias sem contato diário com a água, mostrando se tratar de casos atípicos de contaminação pelo zoósporo, como relatado por Utra Vaz et al. (2009). No referido estudo, ocorreu o acometimento da cavidade nasal de um equino, sendo a primeira descrição pela contaminação atípica por meio da água de bebida disponível na baia. Ao penetrar o tecido cutâneo, o pitium pode atingir órgãos internos e também os ossos, dificultando o processo de tratamento e recuperação. 0 sistema imunológico do tecido ósseo não apresenta a mesma capacidade de resposta dos demais tecidos corporais, possuindo uma menor eficiência circulatória ao conduzir mediadores e células inflamatórias ao periósteo e ao córtex ósseo (Frey Jr et al., 2007). No presente relato, nenhum dos animais apresentou comprometimento ósseo decorrente da lesão cutânea desencadeada pelo pitium.

Neste estudo, as lesões cutâneas possuíam no máximo 60 dias de evolução e tamanho médio de 15 $\mathrm{cm}$. Tais fatores podem ter facilitado a recuperação dos animais. Feridas crônicas geralmente estão associadas a lesões ósseas e levam os animais a óbito ou à necessidade de eutanásia (Maciel et al., 2008). Um estudo realizado no Rio Grande do Sul descreveu que os equinos acometidos pelo pitium apresentaram período de evolução das lesões de
35 dias a vários meses, e que os animais com maior tempo de evolução apresentaram emagrecimento progressivo e desenvolveram ulcerações associadas ao abundante tecido de granulação na pele, fatores que prejudicaram sua recuperação (Assis-Brasil et al., 2015). 0 bom escore corporal dos animais também pode ter contribuído para o processo de recuperação e para a resposta positiva ao tratamento imposto, dada sua influência na resposta do organismo frente ao pitium (Sallis et al., 2003). Animais subnutridos apresentam baixa capacidade de resposta imunológica, facilitando o desenvolvimento do fungo (Miller e Campbell, 1982; Maciel et al., 2008).

A realização do diagnóstico gestacional se deu à farmacologia dos fármacos escolhidos para o tratamento da pitiose. O iodeto de potássio, quando utilizado em éguas durante a gestação, pode desencadear hipotireoidismo congênito no feto (Stewart et al., 2009). Em animais adultos, como relatado por Dória et al. (2008), um animal apresentou hipotireoidismo iatrogênico, ocorrendo a remissão da alteração endócrina após a suspensão do uso do medicamento. Segundo a Direção-Geral de Alimentação e Veterinária de Portugal (DGAV, 2015), quando administrado em éguas gestantes no terço final da gestação, o acetato de triancinolona pode originar um parto prematuro seguido de distocia, morte fetal, retenção de placenta e metrites, com diminuição temporária na produção láctea.

Inúmeros métodos de tratamento têm sido descritos para uso em equinos (Santurio et al., 2004). A excisão cirúrgica como tratamento é realizada pela remoção de toda a lesão e área periférica à ferida, constituindo uma ampla margem de segurança (Salomão-Nascimento et al., 2010). Em extremidades de membros esse procedimento é inviável, pois o tecido cutâneo dessas regiões dificulta a sutura pós-cirúrgica, levando à cicatrização por segunda intenção (Rodrigues e Luvizotto, 2000). Essa característica determinou a decisão de não realização da exérese cirúrgica das lesões nos casos descritos.

A escolha do tratamento com o acetato de triancinolona associado ao iodeto se baseou em estudos realizados por Cardona-Àlvarez et al. (2017). 0 acetato de triancinolona possui propriedades anti-inflamatórias, imunológicas e antialérgicas. Promove o retardo na migração dos leucócitos polimorfonucleares para as feridas, acarretando, 
desta maneira, uma diminuição na fibrinogênese e reduzindo, consequentemente, a reação inflamatória. 0 iodeto de potássio, por sua vez, atua na proteção da glândula tireóide, na interação com as células do sistema imunológico e na ação direta contra agentes infecciosos. Exerce seu efeito antiinflamatório suprimindo a produção, pelos leucócitos polimorfonucleares, de metabólitos tóxicos de oxigênio, inibe a quimiotaxia de neutrófilos e atua na ação dos fagócitos (Costa et al., 2012). 0 efeito anti-inflamatório dos medicamentos e a atuação no sistema imunológico levou à diminuição das feridas ao longo do tratamento, reduzindo o prurido.

\section{Conclusão}

O sucesso no tratamento da pitiose é influenciado pelo tamanho, tempo e local das lesões, além do estado fisiológico do animal. 0 tratamento com acetato de triancinolona e iodeto de potássio se mostrou eficaz, sem que houvesse a necessidade de intervenção cirúrgica e/ou repetição do protocolo. Assim sendo, tal procedimento deve ser considerado como boa alternativa no tratamento de pitiose equina.

\section{Referências}

Assis-Brasil ND, Marcolongo-Pereira C, Stigger AL, Fiss L, Santos BL, Coelho ACB, et al. Equine dermatopathies in southern Brazil: a study of 710 cases. Cienc Rural. 2015;45(3):519-24.

Biava JS, Ollhoff DR, Gonçalves RC, Biondo AW. Zigomicose em equinos -Revisão. Rev Acad. 2007;5(3):225-30.

Cardona-Álvarez J, Vargas-Vilória M, Patarroyo-Salcedo J. Pythiosis cutânea en equinos tratados con acetonida de triamcinolona. Parte 2. Descripción histológica e histoquímica. Rev MVZ Cordoba. 2017;22(1):5638-52.

Carvalho ECQ Lemos LS, Reis Jr JL. Pitiose eqüina: o popular formigueiro da Baixada Campista. Bol Tec UENF. 2000;1:1-48.
Costa LRR. Pythiosis. In: Wilson DA. Clinical Veterinary Advisor: The Horse. 1 ed. St. Louis: Elsevier Saunders; 2012. p. 485-7.

Direcção-Geral de Alimentação e Veterinária (DGAV). Resumo das características do medicamento. Última aprovação dos textos. Lisboa: DGAV; 2015. p. 1-19.

Dória RGS, Canola PA, Ribeiro G, Di Filippo PA, Dias DPM, Valadão CAA. Hipotireoidismo iatrogênico em equino decorrente de excesso de iodo: relato de caso. Arq Bras Med Vet Zootec. 2008;60(3):521-4.

Dória RGS, Freitas SH, Linardi EL, Mendonça FS, Arrud LP, Boabaid FM, et al. Treatment of pythiosis in equine limbs using intravenous regional perfusion of amphotericin B. Vet Surg. 2012;41(6):759-65.

Frey Jr F, Velho JR, Lins LA, Nogueira CEW, Santúrio JM. Pitiose equina na região sul do Brasil. Rev Port Cienc Vet. 2007;102(561-562):107-11.

Maciel ICF, Silveira JT, Maia CA, Sousa RM, Oliveira NJF, Duarte ER. Pitiose fatal em equino tratado inicialmente para habronemose cutânea. Acta Sci Vet. 2008;36(3):293-7.

Marcolongo-Pereira C, Sallis ESV, Raffi MB, Pereira DIB, Hinnah FL, Coelho ACB, et al. Epidemiologia da Pitiose equina na Região Sul do Rio Grande do Sul. Pesq Vet Bras. 2012;32(9):865-8.

Miller RI, Campbell RSF. Immunological studies on equine phycomycosis. Aust Vet J. 1982;58(6):227-31.

Mosbah E, Karrouf GIA, Younis EA, Saad HS, Ahdy A, Zaghloul AE. Diagnosis and surgical management of pythiosis in draft horses: report of 33 cases in Egypt. J Equine Vet Sci. 2012;32(3):164-9.

Rodrigues CA, Luvizotto MCR. Zigomicose e pitiose cutânea em equinos: diagnóstico e tratamento. Rev Ed Contin. 2000;3(3):3-11.

Sallis ESV, Pereira DIB, Raffi MB. Pitiose cutânea em equinos: 14 casos. Cienc Rural. 2003;33(5):899-903. 
Salomão-Nascimento RB, Frazão-Teixeira E, Oliveira FCR. Avaliação hepática e renal em equinos com pitiose tratados com iodeto de potássio, através da determinação das proteínas, substâncias nitrogenadas e enzimas séricas. Rev Bras Med Vet. 2010;32(2):105-10.

Santos CEP, Santurio JM, Marques LC. Pitiose em animais de produção no Pantanal Matogrossense. Pesq Vet Bras. 2011;31(12):1083-9.

Santurio JM, Alves SH, Pereira DB, Argenta JS. Pitiose: uma micose emergente. Acta Sci Vet. 2006;34(1):1-14.

Santurio JM, Catto JB, Camastri Filho JA, Leal AT, Leal ABM. Ferida da moda: epidemiologia, diagnóstico, tratamento e experiência com equinos infectados no Pantanal. Comunicado Técnico 34. Corumbá, MS: Embrapa Pantanal; 2004. 8 p.
Stewart AJ. Respiratory Fungal Infections. In: Robinson NE, Sprayberry KA. Current Therapy in Equine Medicine. 6 ed. Missouri: Saunders; 2009. p. 307-12.

Utra Vaz BB, Maia FCL, Rocha NS, Thomassian A. Pitiose nasal em equino. Med Vet (Recife). 2009;3(4):27-32.

Viana IS, Coutinho IS, Gobbi FP, Gaiotte DG, Graca FAS, Costa APD, et al. Pitiose equina: descrição de 30 casos atendidos na universidade estadual do norte fluminense "Darcy Ribeiro", Campos dos Goytacazes, RJ. VII Simpósio Internacional do Cavalo Atleta; 23-25 abr 2015; Belo Horizonte, MG. Belo Horizonte: V e Z em Minas, Suplemento Especial; 2015. p. 69-70. 